\title{
Two-dimensional binary clusters in a hard-wall trap: Structural and spectral properties
}

\author{
Wen Yang, ${ }^{1,2}$ Minghui Kong, ${ }^{3, *}$ M. V. Milošević, ${ }^{4,5}$ Zhi Zeng, ${ }^{1}$ and F. M. Peeters ${ }^{4, \dagger}$ \\ ${ }^{1}$ Key Laboratory of Materials Physics, Institute of Solid State Physics, Chinese Academy of Sciences, \\ P. O. Box 1129, Hefei 230031, China \\ ${ }^{2}$ Graduate School of the Chinese Academy of Sciences, Beijing 100049, China \\ ${ }^{3}$ Institute of Plasma Physics, Chinese Academy of Sciences, Hefei, Anhui 230031, China \\ ${ }^{4}$ Departement Fysica, Universiteit Antwerpen, Groenenborgerlaan 171, B-2020 Antwerpen, Belgium \\ ${ }^{5}$ Department of Physics, University of Bath, Claverton Down, BA2 7AY Bath, United Kingdom
}

(Received 6 July 2007; published 24 October 2007)

\begin{abstract}
Within the Monte Carlo formalism supplemented by the modified Newton-Raphson optimization technique, we investigated structural and dynamical properties of two-dimensional binary clusters confined in an external hard-wall potential. Two species of differently charged classical particles, interacting through the repulsive Coulomb force are confined in the cluster. Subtle changes in the energy landscape and the stable cluster configurations are investigated as a function of the total number of particles and the relative number of each of the two particle species. The excitation spectrum and the normal modes corresponding to the ground-state configuration of the system are discussed, and the lowest nonzero eigenfrequency as a measure of the stability of the cluster is analyzed. The influence of the particle mass on the eigenfrequencies and eigenmodes are studied, i.e., we study a binary system of particles with different charge and different mass. Several unique features distinct from a monodisperse system are obtained.
\end{abstract}

DOI: 10.1103/PhysRevE.76.041404

PACS number(s): 82.70.Dd, 36.40.Sx, 52.27.Lw

\section{INTRODUCTION}

In 1934, Wigner predicted [1] that electrons crystallize and form a triangular lattice if the density of the electron gas is lowered beyond a critical value. Over the last decades, such Wigner-like ordering in various systems consisting of a finite number of classical particles became a prominent study object [2-5]. Most of the theoretical studies focused on clusters in external parabolic confinement, similar to the action of a uniform neutralizing background of charges. The case of a fixed positive background charge was addressed in Refs. $[6,7]$. In view of possible experimental realizations, the extreme case of hard-wall confinement was considered [8]. Subsequently, an interesting re-entrant melting behavior was observed for hard-wall confined colloidal particles dissolved in water $[9,10]$, which is absent in a parabolically confined system. Obviously, the radial particle fluctuations can be drastically affected by the lateral confinement, and were found to be responsible for an enhanced locking of adjacent particle rings in Ref. [11]. Reference [12] presented a systematic study of the ground state and normal modes of the two-dimensional (2D) hard-wall clusters, where different interparticle interactions (i.e., Coulomb, dipole, logarithmic, and screened Coulomb) were considered.

Very recently, the scientific focus of both theoretical and experimental studies of finite clusters shifted to two-species systems, e.g., with two types of charged particles, that was motivated by the increased complexity of the phase diagram [13]. The structure and melting of heterogeneous clusters made of singly and doubly charged species with the same mass were studied in Refs. [14,15]. Nelissen et al. [16] stud-

\footnotetext{
*mhkong@cashq.ac.cn

†rancois.peeters@ua.ac.be
}

ied the ground state of a finite size system containing one or two impurities with different charge and mass, which was motivated by the experiment of Ref. [17]. Structure and melting of a binary system were studied theoretically by Ferreira et al. [18,19], and experimentally in Refs. [20,21]. The most recent work [22] extended the analysis from binary to multispecies systems. A universal law was proposed, implying that the particles with similar mass-to-charge ratio tend to populate a common shell, independently of the total particle and species numbers in the system. This result is consistent with earlier predictions (see, e.g., Refs. [18,23].). In particular, Ref. [23] indicated the effect of the identical charge-to-mass ratio on the formation of bicomponent $\mathrm{Cou}-$ lomb bicrystals.

All of the above studies considered binary clusters in a parabolic confining potential. Therefore, in this work, we performed a systemic study of the structure and linear dynamics of the 2D binary system in a hard-wall trap since such study has never been done before. Our study is motivated by the recent experiment of Ref. [24], and the Brownian dynamics simulations of the melting properties of the binary systems reported in Ref. [25]. Here we study in detail the ground-state configurations, eigenfrequencies, and eigenmodes of the cluster, not only for binary mixtures of particles with different charge, but also of different mass.

The paper is organized as follows. In Sec. II, the model and the numerical approach are described. Section III is devoted to the ground-state energy and configurations for clusters with different number of particles. In Sec. IV, the eigenmode spectrum for these clusters is discussed, and the effect of particle mass on the normal modes are presented. Our conclusions are given in Sec. V.

\section{NUMERICAL APPROACH}

We study the properties of a binary two-dimensional (2D) cluster confined in a hard-wall potential. The system consists 
of two different kinds of particles, i.e., $N_{f}$ particles of fixed charge $Q_{f} e$ and fixed mass $M_{f}$, and $N_{v}$ particles of variable charge $Q_{v} e$ and variable mass $M_{v}$. The charge ratio between these two kinds of particles is defined as $C_{r}=Q_{v} / Q_{f}>1$, and the corresponding mass ratio as $M_{r}=M_{v} / M_{f}$. To generalize the problem, we take the interparticle interaction proportional to $1 / R^{n^{\prime}}$. The Hamiltonian of such a $2 \mathrm{D}$ system is given by

$$
\begin{aligned}
H= & \sum_{i=1}^{N} V\left(R_{i}\right)+\frac{Q_{f}^{2} e^{2}}{4 \pi \varepsilon R_{h i}>j=1} \sum_{\mid}^{N_{f}} \frac{R_{h}^{n^{\prime}}}{\left|\vec{R}_{i}-\vec{R}_{j}\right|^{n^{\prime}}} \\
& +\frac{Q_{v}^{2} e^{2}}{4 \pi \varepsilon R_{h}} \sum_{k>l=1}^{N_{v}} \frac{R_{h}^{n^{\prime}}}{\left|\vec{R}_{k}-\vec{R}_{l}\right|^{n^{\prime}}}+\frac{Q_{f} Q_{v} e^{2}}{4 \pi \varepsilon R_{h}} \sum_{m=1}^{N_{f}} \sum_{n=1}^{N_{v}} \frac{R_{h}^{n^{\prime}}}{\left|\vec{R}_{m}-\vec{R}_{n}\right|^{n^{\prime}}},
\end{aligned}
$$

with the hard-wall confining potential taken as

$$
V(R)= \begin{cases}0 & \text { for } R<R_{h} \\ \infty & \text { for } R \geq R_{h} .\end{cases}
$$

In the above formulas, $N$ denotes the total number of particles $\left(N=N_{v}+N_{f}\right), e$ is the unit of charge, $R_{h}$ is the radius of the circular hard-wall trap, $\varepsilon$ is the dielectric constant of the surrounding medium, and $\vec{R}_{i}=\left(x_{i}, y_{i}\right)$ is the position of the $i$ th particle with $R_{i} \equiv\left|\vec{R}_{i}\right|$. Note from Eq. (1) that the total energy of the studied system does not depend on the mass of the particles in contrast to the case with parabolic confinement potential [18].

We can obtain the Hamiltonian in a dimensionless form if we choose $R_{h}$ as the unit of length, $E_{0}=Q_{f}^{2} e^{2} / 4 \pi \varepsilon R_{h}$ as the unit of energy, and $Q_{f} e$ as the unit of charge. Equation (1) then reads

$$
H=\sum_{i>j=1}^{N_{f}} \frac{1}{\left|\vec{r}_{i}-\vec{r}_{j}\right|^{n^{\prime}}}+\sum_{k>l=1}^{N_{v}} \frac{C_{r}^{2}}{\left|\vec{r}_{k}-\vec{r}_{l}\right|^{n^{\prime}}}+\sum_{m=1}^{N_{f}} \sum_{n=1}^{N_{v}} \frac{C_{r}}{\left|\vec{r}_{m}-\vec{r}_{n}\right|^{n^{\prime}}},
$$

with the hard-wall potential in the form

$$
V(r)= \begin{cases}0 & \text { for } r<1 \\ \infty & \text { for } r \geq 1\end{cases}
$$

Regarding the interparticle interaction, in this paper we will restrict ourselves to the Coulomb repulsion, i.e., $n^{\prime}=1$ in Eqs. (1) and (3).

The ground-state configurations were obtained by minimizing the energy [Eq. (3)] using the Monte Carlo simulation technique, followed by a Newton optimization procedure proposed by Schweigert and Peeters (for details, refer to Ref. [26]). The eigenmode spectrum is then obtained from the eigenvalues and eigenvectors of the dimensionless dynamical matrix with elements

$$
H_{\alpha \beta, i j}=\left.\frac{\partial^{2} H}{\sqrt{m_{i} m_{j}} \partial \alpha_{i} \partial \beta_{j}}\right|_{\alpha_{i}=\alpha_{i}^{0}},
$$

where $\alpha, \beta$ denote one of the Cartesian coordinates $(x, y)$ of the given particle, $\alpha_{i}^{0}$ gives the position of the $i$ th particle in

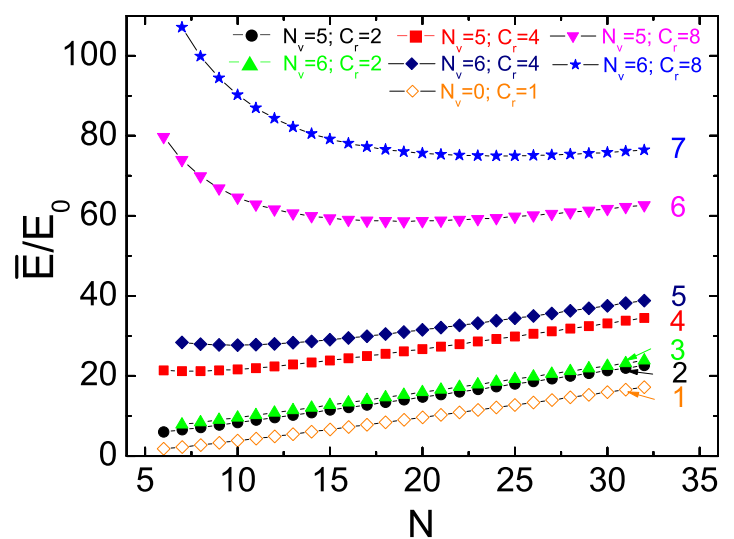

FIG. 1. (Color online) Average energy per particle $\bar{E}$ as a function of the total number of particles $N$, for clusters with $N_{v}=5$ and $N_{v}=6$, and $C_{r}=2,4$ and 8 , respectively. In addition, the result for a monodisperse system $\left(N_{v}=0\right)$ with singly charged particles is presented in comparison.

the ground-state configuration, and $m_{i}=M_{i} / M_{f}$ is the reduced mass of each particle $(i=1,2 \ldots N)$. Therefore $m_{i}$ equals $M_{r}$ for particles with varied mass $M_{v}$ and 1 for particles with fixed mass $M_{f}$. The eigenfrequencies in this paper will be expressed in units of $\omega^{\prime}=\sqrt{E_{0} /\left(M_{f} R_{h}^{2}\right)}$.

\section{STRUCTURE OF THE BINARY CLUSTER IN THE GROUND STATE}

In this section, we will consider the ground-state configurations and the corresponding energy of the studied system. In Fig. 1, we show the average energy per particle in the ground state $\bar{E}$, for clusters with different total number of particles $N$. Each line in Fig. 1 corresponds to a system with different values of $N_{v}$ and $C_{r}$. For comparison, we also show the result for a monodisperse system with singly charged particles (line 1, $N_{v}=0$ and $C_{r}=1$ ). The other curves are for different charge of $N_{v}=5$ or 6 particles (i.e., different $C_{r}$ ) and we increased the number of low-charged particles. Here we show an essentially important result-with increasing charge of $N_{v}$ particles, as we add low-charged particles to the system (i.e., increase the total number of particles $N$ ), the energy $\bar{E}$ first decreases and then increases. This is depicted by curves 4-7 in Fig. 1, which all decrease first and then rise, while the lower three curves 1-3 obtained for $C_{r}=1$ and $C_{r}$ $=2$ monotonically increase. Therefore for each of the four upper lines (4-7) we can obtain the optimal number of particles in the cluster $N_{L}$, which provides the lowest average energy per particle. We find that, for $N_{v}=5, N_{L}$ equals 7 for $C_{r}=4$, while it increases to $N_{L}=19$ for $C_{r}=8$. Similarly, for $N_{v}=6, N_{L}$ increases from 10 to 24 for $C_{r}=4$ and 8, respectively.

Note that this trend of decrease and subsequent increase of the average particle energy vs the number of particles $\bar{E}(N)$ curve is unusual. For parabolic $[8,27]$ and hard-wall confinement [12], the energy always monotonically increases with the particle number $N$, similarly to curves $1-3$ in Fig. 1 . 

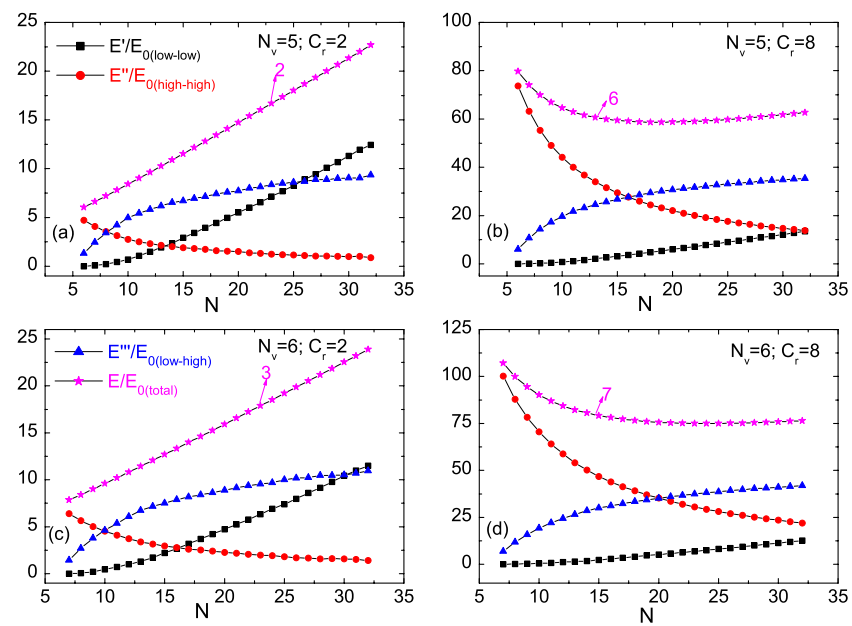

FIG. 2. (Color online) (a) The different energy contributions for $N_{v}=5$ and $C_{r}=2$ clusters, as a function of the total number of particles $N$. The line with black squared dots gives the average interaction energy $E^{\prime}$ between low-charged particles, the red dotted line shows the average interaction energy $E^{\prime \prime}$ between high-charged particles, the blue triangles correspond to the average interaction energy $E^{\prime \prime \prime}$ exclusively between low and high charged particles, and the purple pentagons indicate the total energy per particle $\bar{E}$. The same notation is used in (b), (c), and (d) for different parameter values.

In what follows, we clarify the unusual $\bar{E}(N)$ behavior of binary clusters. The total energy $E$ for binary systems consists of three parts: averaged interaction energy $E^{\prime}$ (low-low) between low-charged particles, averaged interaction energy $E^{\prime \prime}$ (high-high) between particles with higher charge, and averaged interaction energy $E^{\prime \prime \prime}$ (low-high) between the two species. In Fig. 2, we show the dependence of all latter energies on the number of particles in the cluster $\left[E(N), E^{\prime}(N)\right.$, $\left.E^{\prime \prime}(N), E^{\prime \prime \prime}(N)\right]$, for $N_{v}=5,6$ and $C_{r}=2,8$. As shown in Figs. 2(a)-2(d), $E^{\prime}$ (black squares) and $E^{\prime \prime \prime}$ (blue triangles) increase when $N$ increases while $E^{\prime \prime}$ (red dots) always decreases. Obviously, the decrease of $E^{\prime \prime}(N)$ is the source of the decreasing average energy per particle in small clusters. Being the interaction energy between a fixed number of $N_{v}$ particles (in our case 5 or 6$), E^{\prime \prime} / N$ must decrease with increasing $N$. As three aforementioned energies constitute the total energy $E$, the decrease of $E^{\prime \prime}(N)$ competes with the increase of $E^{\prime}(N)$ and $E^{\prime \prime \prime}(N)$ to determine the final tendency of $E(N)$. Consequently, when the charges of $N_{v}$ particles are much larger than those of the other particles, the contribution of the interaction energy between $N_{v}$ particles dominates. E.g. in line 6 of Fig. 1 [see Fig. 2(b)], when $C_{r}$ is large, $E^{\prime \prime}$ is much larger than $E^{\prime}$ and $E^{\prime \prime \prime}$ for the total number $N<15$. As a result, the averaged energy $E$ decreases when $N<N_{L}$. For sufficiently large $N$, the slowly decreasing $E^{\prime \prime}$ is eventually overpowered by the increasing tendency of $E^{\prime}$ and $E^{\prime \prime \prime}$.

Next, we show the investigated ground-state configurations for binary systems with fixed number of high-charged particles $N_{v}$ while increasing the number of low-charged particles. Figure 3 shows characteristic lowest energy configurations we obtained for clusters with $N_{v}=5$ and 6 , and $C_{r}$
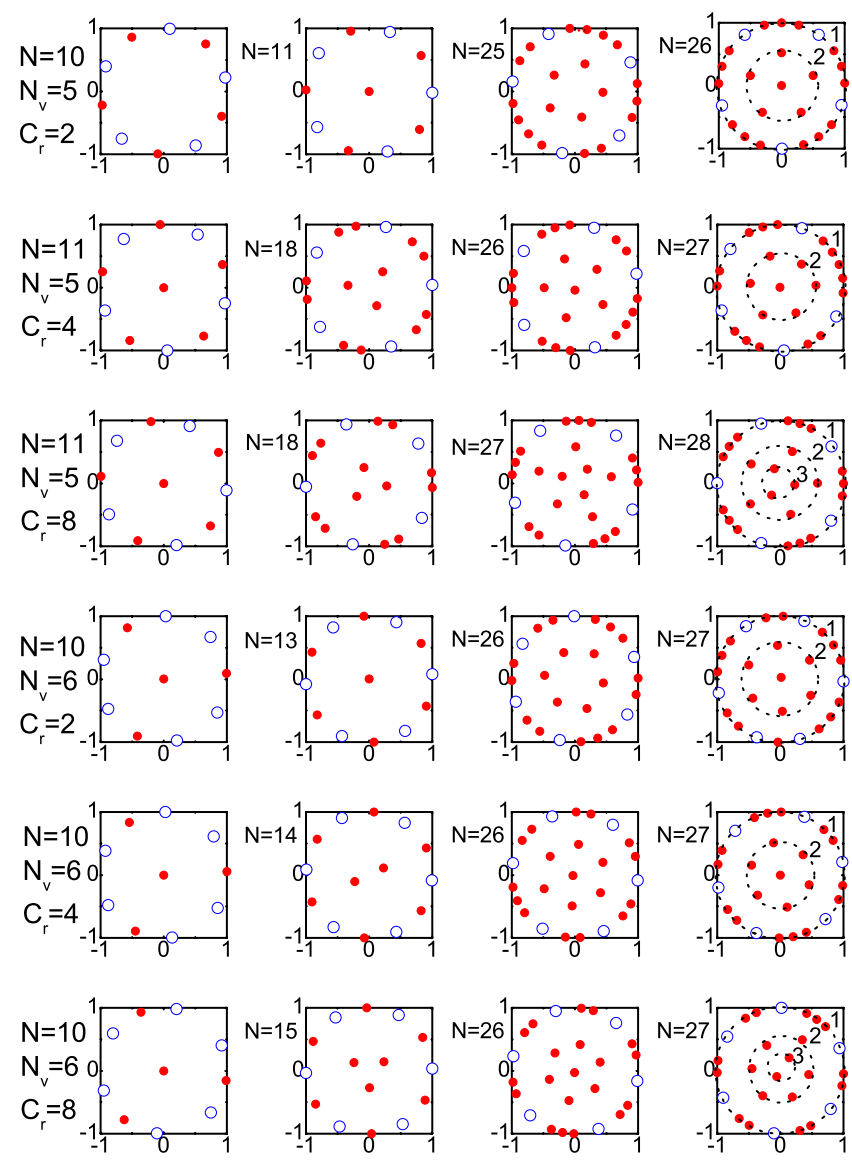

FIG. 3. (Color online) Typical GS configurations for binary clusters with $N_{v}=5$ and $6, C_{r}=2,4$, and 8 , respectively. The total number $N$ is marked on the left upper corner of each figure. The clusters shown in each row have identical values of $N_{v}$ and $C_{r}$. Open circles denote the high-charged particles while the full dots refer to the low-charged particles. The dotted circles and the corresponding numbers in the fourth column indicate the shell structure of the obtained clusters.

$=2,4$, and 8 , shown in respective rows. One should note that all the high-charged particles (open dots) in our system always reside at the rim of the cluster, which is analogous to the case of binary systems confined in a parabolic trap [14]. At the same time, few added low-charged particles (full dots) are located in between high-charged particles at the hard wall. Dotted circles in some figures of Fig. 3 emphasize the shell structures. Those are hardly surprising but still differ from previous works $[8,12]$ that studied typical shell structures for monodisperse systems. Namely, chosen parameters $N_{v}$ and $C_{r}$ strongly affect the ground-state structure of the cluster. By comparing configurations in the upper three rows $\left(N_{v}=5\right)$ with those in the bottom three rows $\left(N_{v}=6\right)$, one finds, e.g., that the inner shell presents a pentagonlike structure when $N_{v}=5$ while it exhibits a hexagonlike structure when $N_{v}=6$. This feature is driven by the energy minimization of the present Coulomb repulsions when the inner shell of low-charged particles matches the structure of highcharged particles in the outer shell. This brings us to the new definition of so-called "magic" numbers: as in binary clusters the inner shell tends in most of the cases to be occupied by 
TABLE I. Shell structures [denoted by $\left(N_{1}, N_{2}, \ldots\right)$ ] of the ground-state configurations for a monodisperse system $\left(N_{v}=0\right)$ and binary systems with $N_{v}=5,6$ and $C_{r}=2,4$, and 8, respectively.

\begin{tabular}{|c|c|c|c|c|c|c|c|}
\hline \multirow[b]{2}{*}{$N$} & \multirow[b]{2}{*}{$N_{v}=0$} & \multicolumn{3}{|c|}{$N_{v}=5$} & \multicolumn{3}{|c|}{$N_{v}=6$} \\
\hline & & $C_{r}=2$ & $C_{r}=4$ & $C_{r}=8$ & $C_{r}=2$ & $C_{r}=4$ & $C_{r}=8$ \\
\hline 6 & 6 & 6 & 6 & 6 & 6 & 6 & 6 \\
\hline 7 & 7 & 7 & 7 & 7 & 7 & 7 & 1,6 \\
\hline 8 & 8 & 8 & 8 & 8 & 8 & 8 & 1,7 \\
\hline 9 & 9 & 9 & 9 & 9 & 9 & 1,8 & 1,8 \\
\hline 10 & 10 & 10 & 1,9 & 1,9 & 1,9 & 1,9 & 1,9 \\
\hline 11 & 11 & 1,10 & 1,10 & 1,10 & 1,10 & 1,10 & 1,10 \\
\hline 12 & 1,11 & 1,11 & 1,11 & 1,11 & 1,11 & 1,11 & 2,10 \\
\hline 13 & 1,12 & 1,12 & 1,12 & 2,11 & 1,12 & 2,11 & 2,11 \\
\hline 14 & 1,13 & 1,13 & 2,12 & 2,12 & 1,13 & 2,12 & 2,12 \\
\hline 15 & 1,14 & 2,13 & 2,13 & 2,13 & 2,13 & 2,13 & 3,12 \\
\hline 16 & 1,15 & 2,14 & 3,13 & 3,13 & 2,14 & 3,13 & 3,13 \\
\hline 17 & 2,15 & 2,15 & 3,14 & 3,14 & 3,14 & 3,14 & 4,13 \\
\hline 18 & 2,16 & 3,15 & 3,15 & 3,15 & 3,15 & 4,14 & 4,14 \\
\hline 19 & 3,16 & 3,16 & 4,15 & 4,15 & 3,16 & 4,15 & 5,14 \\
\hline 20 & 3,17 & 3,17 & 4,16 & 4,16 & 4,16 & 4,16 & 5,15 \\
\hline 21 & 3,18 & 4,17 & 4,17 & 5,16 & 4,17 & 5,16 & 5,16 \\
\hline 22 & 4,18 & 4,18 & 5,17 & 5,17 & 4,18 & 5,17 & $1,5,16$ \\
\hline 23 & 4,19 & 5,18 & 5,18 & $1,5,17$ & 5,18 & $1,5,17$ & $1,5,17$ \\
\hline 24 & 4,20 & 5,19 & $1,5,18$ & $1,5,18$ & 5,19 & $1,5,18$ & $1,6,17$ \\
\hline 25 & 5,20 & 5,20 & $1,5,19$ & $1,5,19$ & 5,20 & $1,6,18$ & $1,6,18$ \\
\hline 26 & 5,21 & $1,5,20$ & $1,5,20$ & $2,5,19$ & 6,20 & $1,6,19$ & $1,6,19$ \\
\hline 27 & 5,22 & $1,5,21$ & $1,6,20$ & $3,5,19$ & $1,6,20$ & $1,6,20$ & $2,6,19$ \\
\hline 28 & 6,22 & $1,6,21$ & $1,6,21$ & $3,5,20$ & $1,6,21$ & $2,6,20$ & $3,6,19$ \\
\hline 29 & 6,23 & $1,6,22$ & $1,7,21$ & $4,5,20$ & $1,6,22$ & $2,6,21$ & $3,6,20$ \\
\hline 30 & $1,6,23$ & $1,7,22$ & $1,7,22$ & $4,5,21$ & $1,7,22$ & $3,6,21$ & $4,6,20$ \\
\hline
\end{tabular}

$N_{v}$ low-charged particles. This is particularly important for the first shell, as it has been shown previously for parabolic confined classical clusters [8,27] (and confirmed also for superconducting vortices in disks $[28,29])$ that the number of particles in the innermost shell cannot exceed 5. For monodisperse clusters in a hard wall [12], the corresponding threshold number equals 6 . In our system this restriction mostly depends on the values of $N_{v}$ and $C_{r}$, which is evident for $C_{r}>2$.

As another structural difference from monodisperse clusters, as shown in Fig. 3, our clusters with more high-charged particles (especially for higher $C_{r}$ ) have more inner lowcharged particles for fixed $N$, due to the increased repulsion from the cluster edge where high-charged particles reside. Note also that our binary systems have by far a more complex structure than the monodisperse ones; the ground-state configurations are rather complicated and are determined by several competing interactions between different particle species, within each species itself, and all together with the confinement. In essence, parameters $N, N_{v}$, and $C_{r}$ all together determine the concrete configuration of a given binary system.
As explained above, for small $N$, particles first occupy the edge positions at the hard wall due to the mutual Coulomb repulsion. Added low-charged particles first fill up the outer shell and then are gradually pressed into the interior of the cluster where they form a new shell. In a similar case, for a sufficient number of low-charged particles, they can be redistributed further inside into a third shell. Table I shows the packing sequences of the ground-state configurations for $N$ $=6-30$ clusters with $N_{v}=5,6$ and $C_{r}=2,4$, and 8, respectively. Each configuration is indicated by the number of particles in different shells, starting from the innermost one. In Table I, we show directly the manner of shell-filling, when each new shell appears, and how parameters $N_{v}$ and $C_{r}$ affect the concrete formation of particular configurations.

Let us briefly discuss configurational properties of clusters with five doubly charged particles $\left(N_{v}=5, C_{r}=2\right)$. For small $N \in\{6-10\}$, all the particles are situated at the edge of the wall. When a critical number of particles at the edge is achieved $(N=11)$, the inner shell is formed. For $N \in\{11$ $-25\}$, the system has a two-shell structure. When the number of particles in the second shell reaches 5 , a third shell is created; this holds on for $N \in\{26-30\}$. On the other hand, for $N_{v}=5$ and $C_{r}=4$, the second shell appears after $N=10$. 

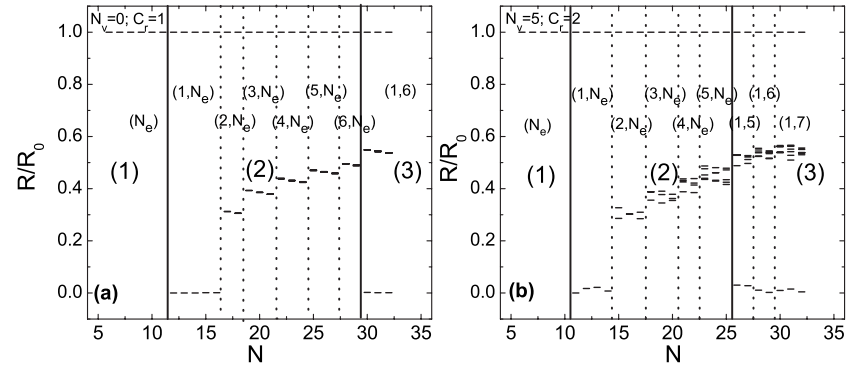

FIG. 4. (a) Radii of all the particles as a function of the total number of particles $N$ for the monodisperse system. (b) Similar to (a), but for binary clusters with $N_{v}=5$ and $C_{r}=2$. Solid vertical lines separate the clusters with different number of shells, while dotted vertical lines show finer configurational transitions; numbers in the brackets give the exact shell structure of the clusters between the dotted lines, where $N_{e}$ is the number of particles situating at the edge of the cluster.

The critical number of particles in the second shell is again 5 , thus the third shell forms after $N=24$. For $C_{r}=8$, the same pattern continues, so we can conclude that new shells are created at smaller $N$ as $C_{r}$ is increased for fixed number of high-charged particles.

A higher number of high-charged particles $N_{v}$ also influences the cluster structure. In the case of $N_{v}=6$ and $C_{r}=2$ systems, $N=10$ and $N=27$ are the critical points when the new shells are created, and for $C_{r}=4$, these drop to $N=9$ and $N=23$ (and $N=7$ and $N=22$ for $C_{r}=8$ ). Therefore, increasing the number of high-charged particles $N_{v}$ has an additive effect to the one after increasing charge $C_{r}$.

The parameter $C_{r}$ can also have an important impact on the value of the critical number. As shown in Table I, the critical number of the second shell is 5 for both cases of $N_{v}=6$ and $C_{r}=4$ or 8 . Compare this result with the case of $N_{v}=6$ and $C_{r}=2$, the increased repulsive force may be the key ingredient to hold back the formation of a hexagon. At the same time, for the monodisperse system confined in a hard wall, the critical number of the second shell is 6 [12]. Generally, we can conclude that for a binary system the critical number for the second shell cannot be more than 6 and the actual value is determined by the values of $N_{v}$ and $C_{r}$. When the values of $N_{v}$ and $C_{r}$ are larger, the maximal number of particles in the second shell is smaller.

In Fig. 4(b), the radial distributions of all the particles are plotted as a function of the total number $N$ for clusters with $N_{v}=5$ and $C_{r}=2$, next to those for the monodisperse system shown in Fig. 4(a). According to the number of shells for every cluster, we can divide the diagram into three parts. In part 1, all particles are located at the edge of the wall and form a perfect ring. Particles in every cluster of part 2 form two shells, and the clusters in part 3 have three shells. In areas 2 and 3, the dotted lines separate the clusters with a different number of particles in each shell. For example, $\left(3, N_{e}\right)$ of part 2 indicates that clusters in this area all have two shells with three particles in the inner shell and the remaining $N_{e}$ particles in the outer shell. Between the dotted lines, the radius of the inner shell is expected to decrease as
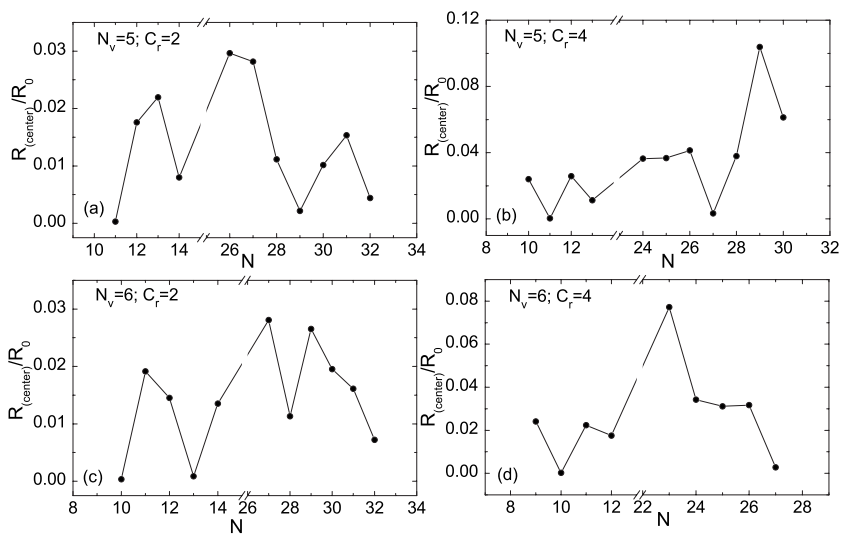

FIG. 5. The off-center position of the central particle in a $\left(1, N_{e}\right)$ cluster as a function of $N$, for different values of the parameters of the binary cluster. Solid lines in figures (a), (b), (c), and (d) are guides to the eye.

the number of particles in the outer shell increases [and consequently the lateral compression on the inner particles increases, see Fig. 4(a)].

However, the radial distribution of particles for binary systems in Fig. 4(b) shows several distinct features. First of all, we find that the first particle that leaves the outer shell is not always located in the center of the cluster. Its displacement from the central position follows from the nonuniform distribution of high-charged particles on the cluster edge for particular numbers of particles in the outer shell. The displacement vs $N$ is shown in Fig. 5 for different parameter values, and can exceed $10 \%$ of the size of the hard-wall trap. For the same reason, the inner shells in areas 2 and 3 of Fig. 4(b) no longer form a perfect ring, contrary to the case of monodisperse systems. In conclusion, the mixing of two kinds of particles in the cluster makes the spatial distribution highly asymmetric. It basically appears impossible for the binary clusters to achieve perfect-shell configurations, as particles distribute in deformed ring structures.

\section{PROPERTIES OF THE EIGENFREQUENCY SPECTRUM AND NORMAL MODES}

In this section, we investigate the specific spectral features of binary clusters, i.e., the unique properties of their excitation spectrum, normal modes, and eigenvectors for the ground-state configurations. It is already known that there exist $2 N$ normal modes for a $N$-particle system. However, in the hard-wall case, as the radial motion of particles at the edge requires large energy, particles at the edge are practically radially immobilized at the wall, and can only move along the perimeter [12]. Consequently, the number of relevant modes for the hard-wall system reduces to $2 N-N_{e}$, with $N_{e}$ being the number of particles in the outer shell. In what follows, our numerical simulations of these $2 N-N_{e}$ modes will be compared to those for the monodisperse clusters reported in Ref. [12].

We plot the full excitation spectrum of the normal modes as a function of total number of particles $N$ for binary clus- 

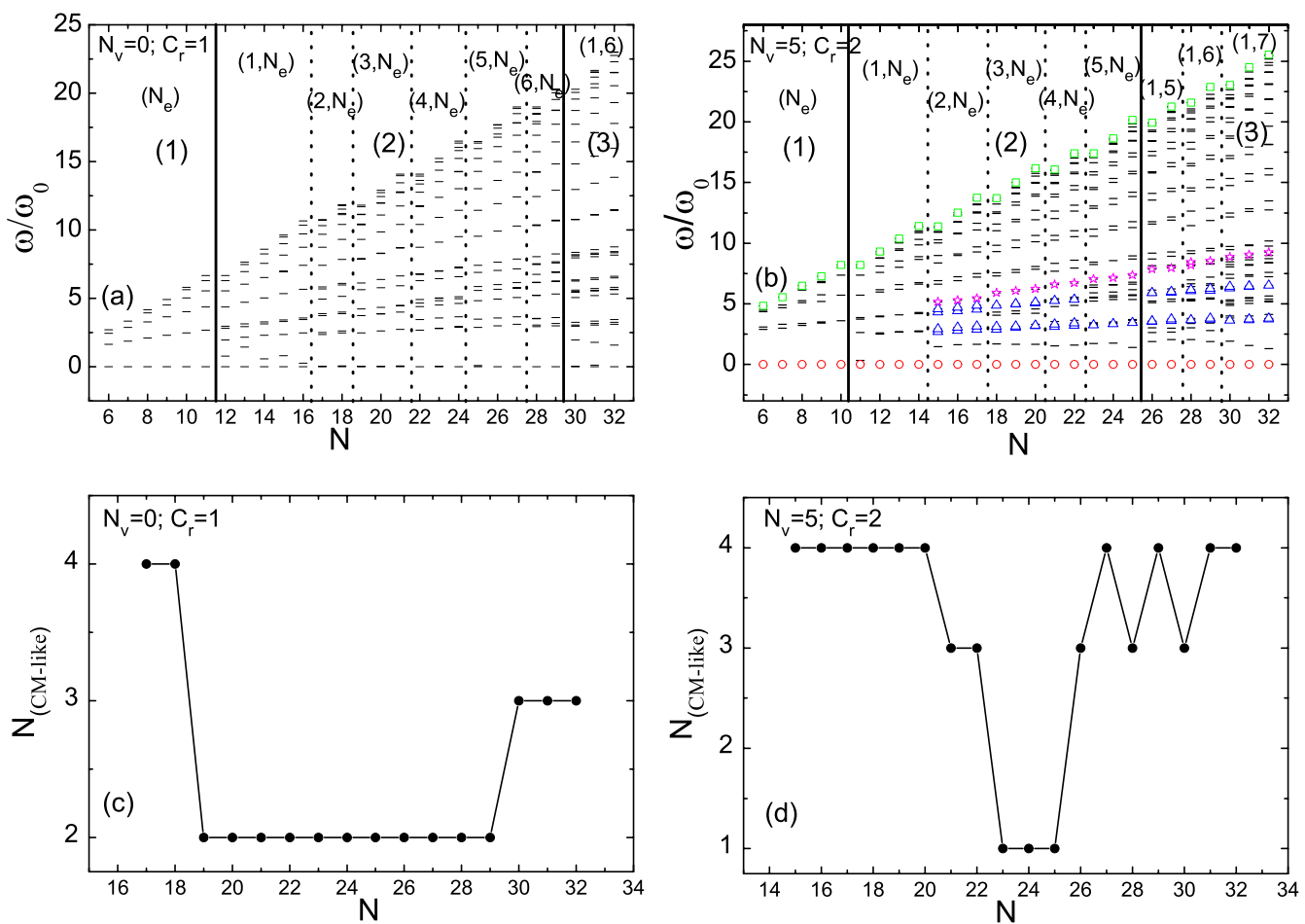

FIG. 6. (Color online) (a) Excitation spectrum of the monodispersive cluster as a function of the total number of particles; (b) same as (a) for the binary cluster with $N_{v}=5$ and $C_{r}=2$. The center-of-mass- and breathing-mode-like, $\omega=0$ and $\omega_{\max }$ modes are marked by triangles, pentagons, circles, and squares, respectively. Figures (c) and (d) show the number of center-of-mass modes as a function of $N$, for the monodisperse and binary cluster, respectively. Solid lines in figures (c) and (d) are guides to the eye.

ters with $N_{v}=5$ and $C_{r}=2$ in Fig. 6(b), next to the same for a monodisperse cluster shown in Fig. 6(a). For Coulomb systems confined in a parabolic trap, three common normal modes can be identified [26,30,31]: rotation as a whole (with frequency $\omega=0)$, the center-of-mass mode $(\mathrm{CM}, \omega=\sqrt{2})$, and the breathing mode $(\mathrm{BM}, \omega=\sqrt{6})$. These three common modes have been found in the three-dimensional systems as well [32]. In the hard-wall case, only $\omega=0$ eigenfrequency remains common for all clusters regardless of $N$ [as shown in Figs. 6(a) and 6(b)]. However, the CM and BM modes still exist, but now with a frequency that depends on $N$. In binary systems, CM and BM modes also exist, which are similar to the $\mathrm{CM}$ and BM modes of the monodisperse system (see Ref. [12]). Due to asymmetry in the interparticle interactions in the binary cluster, these modes show certain irregularities, and are only CM-like and BM-like, as illustrated in vec5, vec6, vec10, vec11, and vec13 vector plots in Fig. 7. Because of the forbidden radial motion of particles in the outer shell, only the inner particles can undertake CM-like or BM-like oscillations (see Fig. 7). Therefore for $N=6-14$ in Fig. 6(b), when the particles are all at the edge or only a single one is in the center, there is no sense of discussing the CM-like or BM-like modes for hard-wall confinement. Starting from $N$ $\geq 15$, with more than one particle in the inner region of the cluster, we can assign some eigenmodes to the CM or BM modes for the particles in the central region. The frequencies for $\omega=0$, CM-like and BM-like modes are marked by different symbols in Fig. 6. Figures 6(c) and 6(d) show the number of identified CM modes as a function of $N$ for the monodisperse and binary system. Note that four CM-like modes were found up to $N=20$ in binary clusters [Fig. 6(d)], and this number strongly differs as a function of $N$ compared to Fig. 6(c). On average, we find a more pronounced quasidegeneracy of the CM-like modes compared to the monodisperse

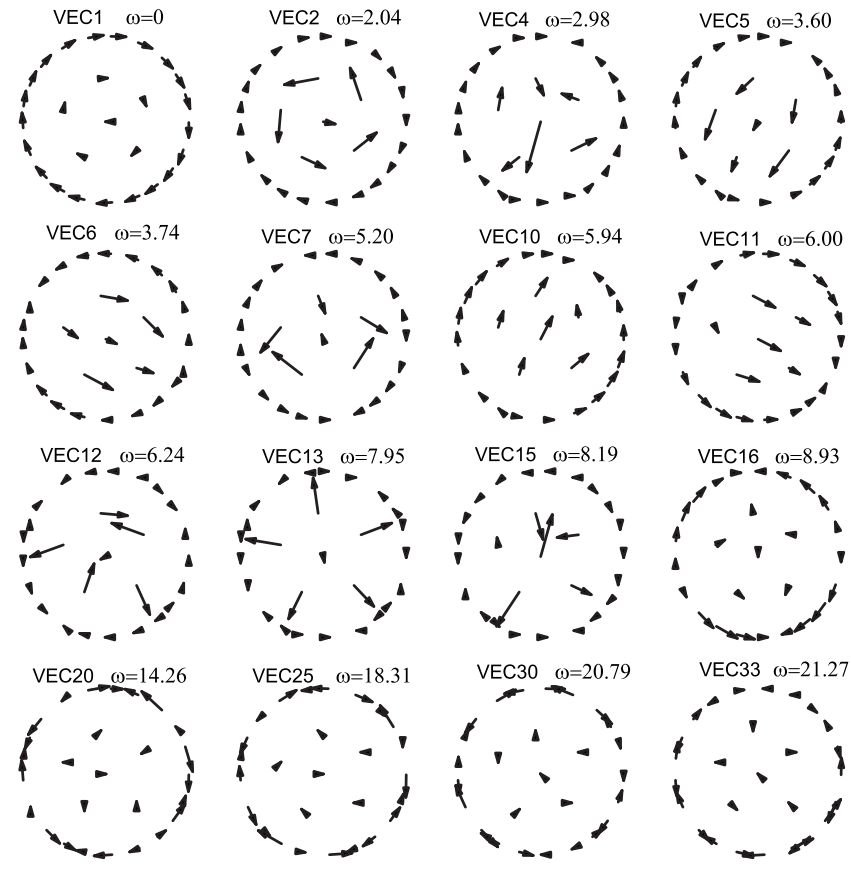

FIG. 7. Characteristic eigenvectors for a binary cluster with $N$ $=27, N_{v}=5$, and $C_{r}=2$. 

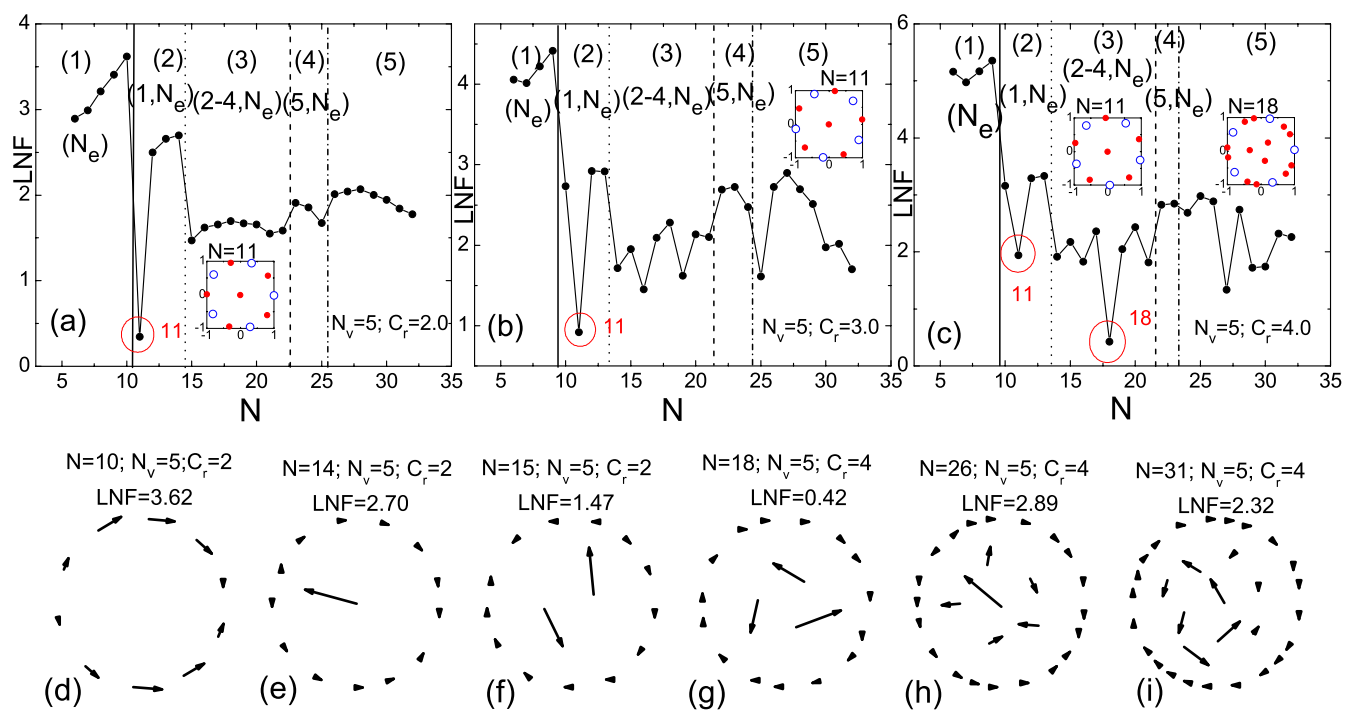

FIG. 8. (Color online) (a)-(c) Present the LNF for $N=6-32, N_{v}=5$, and $C_{r}=2,3$, and 4, respectively. (d)-(f) Eigenvectors of LNF for clusters with $N_{v}=5, C_{r}=2, N=10,14$, and 15, respectively. (g)-(i) Eigenvectors of LNF for clusters with $N_{v}=5, C_{r}=4, N=18,26$, and 31, respectively.

clusters in hard-wall confinement. Note also that latter CM modes are truly degenerate for clusters in radially symmetric traps (parabolic $[18,26]$ or hard wall [12]), while in our binary system they are each identifiable and have distinguishable frequencies.

Next, according to the structural division of Fig. 4(b), Fig. 6(b) can also be separated into corresponding areas. As explained above, the clusters in each region [between two vertical lines in Fig. 4(b)] have the same inner shell structure. As shown in Fig. 6(b), the corresponding eigenfrequencies in each particular region exhibit a similar distribution, while slightly rising in value with increasing $N$. However, at a crossover to the next region, the highest eigenfrequency [marked by squares in Fig. 6(b)] remains virtually unchanged. Namely, at those values of $N$, an additional particle in the cluster is added to the interior of the cluster and the outer shell remains unchanged. As the highest frequency corresponds to the dynamics of the outer shell, it remains the same for clusters with $N$ and $N+1$ particles. For example, when $N$ increases, the ascending trend of $\omega_{\max }$ is evident up to $N=11$, where the value of $\omega_{\max }$ remains nearly the same, i.e., $\omega_{\max }$ of the $N=11$ cluster is almost equal to the one of the $N=10$ cluster. In Fig. 6(b), such a feature is found as well in clusters with $N=15,18,21,23,26$, etc., and each corresponds to structural transitions from Fig. 4(b). In Fig. 7, the higher energy excitations for the case of $N=27\left(N_{v}=5, C_{r}\right.$ $=2$ ) are plotted from vec16 to vec 33 . For these vector plots, especially the vec 33 of $\omega_{\max }$, outer shell particles move in pairs and oscillate toward each other, while particles on inner shells oscillate with far lower amplitude. Hence $\omega_{\max }$ excitation is mostly determined by the oscillation of particles at the edge, and the corresponding eigenfrequency remains approximately the same for an unchanged number of particles in the outer shell $N_{e}$. At the same time, for the lower energy excitations shown in Fig. 7, the inner particles oscillate strongly while the outer ones are frozen.
Among all of the eigenfrequencies, the lowest nonzero eigenfrequency (LNF) has stirred special interest in the scientific community as it presents a measure for the stability of the ground state. Figures $8(\mathrm{a})-8$ (c) present the LNF for $N$ $=6-32, N_{v}=5$, and $C_{r}=2,3$, and 4, respectively. In Fig. 8(a) we show a clear ladder shape of the $\operatorname{LNF}(N)$ curve. To emphasize the hierarchy of structures along $\operatorname{LNF}(N)$ curves in Fig. 8, we indicate structural transitions by vertical lines. We divide each diagram into five parts with distinct behavior of LNF. For clusters within part 1 marked as $\left(N_{e}\right)$, all particles are on a single ring at the edge. In part 2 of $\left(1, N_{e}\right)$, only one low-charged particle sits in the central region. The two-shell clusters with more than one particle in the inner shell constitute parts 3 and 4, with the latter having $N_{v}$ (five) lowcharged particles in the inner shell. In part 5 of each diagram in Fig. 8, three shells are formed in each cluster.

For reasons already explained earlier, the LNF is found to be largest in region 1, as particles in the outer shell are hardly subjected to any dynamics. The LNF in region 2 decreases, as the oscillations in the outer shell are stimulated by shorter range interaction with the central particle. The descending trend of LNF continues in region 3. For single-shell clusters, the LNF mode corresponds to the two halves of the a ring moving in opposite directions [such as the case in Fig. 8(d)], whereas for double-shell clusters the LNF mode mostly involves oscillations of the inner shell [Figs. $8(\mathrm{e})-8(\mathrm{i})$ ]. It is easier to excite one inner particle of the double-shell clusters in regions 2 and 3 than to excite the outer particles in region 1 . Thus it is easily conceived that the single-shell clusters are more stable than the double-shell clusters. This also explains the difference of LNF between the regions 2 and 3 as more particles in the central ring are easily excited compared to the single particle case. As shown in Figs. 8(e), the LNF mode for clusters in region 2 still has the two halves of the outer shell moving in opposite direc- 
tions. In region 3, the LNF mode corresponds to the rotation of the inner particles, as shown in Figs. 8(f) and 8(g).

Following this tendency, one expects the LNF to decrease further for double-shell clusters with more inner particles. However, we find that LNF increases in region 4 for five inner particles. This is clearly related to the "matching" of the inner shell to the configuration of the $N_{v}$ high-charged particles in the outmost shell. Namely, five low-charged particles form a pentagon inside the cluster, rotated with $\pi / 5$ angle with respect to the pentagon formed by five highcharged particles sitting at the edge. Because of this commensurability effect, this configuration is difficult to excite. This and similar structures we classified as matching configurations.

With further enlargement of clusters, new shells are created. In region 5 of Figs. 8(a)-8(c) we show LNF for tripleshell clusters. The variation of LNF with increased $N$ in this area is somewhat similar to regions $2-4$. First, only one particle sits in the center which resembles the cases in region 2. When more particles are pushed in the center, the formation of the inner shells mimics the ones from regions 3 and 4 . These facts only emphasize the effect of the center-shell configuration on the lowest nonzero excitation. However, it should be noted that LNF seems to decrease as $N$ is further increased in region 5. Namely, the individual interparticle interaction is gradually replaced by the effective interactions between the shells as a whole, which results in the stimulated intershell rotation, weakens the stability, and decreases the LNF.

Let us now discuss the distinct, lowest LNF points found in some structural regions, marked by red circles and corresponding configurations in Fig. 8. A common feature for all clusters of these peculiar points is that the high-charged particles and the low-charged particles are alternately placed at the edge while remaining low-charged particles form a radially symmetric inner shell in the center. The inner shell of low-charged particles experiences a symmetric potential, which can easily excite collective motions of inner particles, especially rotation. Intuitively, it is much easier to excite collective motions of regular clusters than asymmetrical motions of nonuniform clusters. Consequently, uniform structures have lower LNF. We should emphasize that these uniform configurations are distinct from the previously discussed matching configurations. The uniform configurations require a uniform distribution of high-charged and lowcharged particles at the edge, while the matching configurations stress the same symmetry of the low-charged particles in the inner shell and the high-charged particles in the outer shell. In the present case, for clusters at the red circled points in Fig. 8, the corresponding configurations are uniform but not matching, and they are easier for excitation than matching structures. If the cluster is both uniform and matching, such as the one for $N=26\left(N_{v}=5, C_{r}=2\right)$ shown in Fig. 3, the corresponding LNF is high [see Fig. 8(a)]. Therefore matching is more deterministic for the stability of the cluster than the uniform structure.

In Ref. [12], LNF vectors of the small and large monodisperse Coulomb clusters confined in a hard-wall trap were studied. It was found that once there is more than one par- ticle at the center, the LNF mode always corresponds (even for large systems) to the rotation of all central particles as a whole in the direction opposite to the one for the particles at the edge, so-called intershell rotation. In our case of multishell binary clusters, the LNF modes are richer than for monodisperse systems. As shown in Figs. 8(g)-8(i), the eigenvectors of the LNF for some binary systems with $N_{v}$ $=5$ and $C_{r}=4$ correspond to intershell rotation, and others to vortexlike rotation, or several rotations in different parts of the cluster. Contrary to the simple LNF mode for monodisperse systems [12], the diversified LNF eigenvectors of binary systems are induced by the spatially inhomogeneous forces of the outer shell acting on the inner particles.

All above studied binary clusters consisted of particles with different charge but equal mass. In what follows, we briefly discuss the properties of the binary systems with two kinds of particles-with different mass and different charge. One should note that changing the mass of particles has no influence on the total energy and ground-state configurations of the system, as mass does not influence the interparticle interactions or the hard-wall confinement. Therefore we restrict the present discussion to the effect of particle mass on the LNF modes. In Figs. 9(a)-9(d), we show the LNF for $N=6-32$ with $N_{v}=4-7$ and $C_{r}=2$. The reduced mass of lowcharged particles $m_{f}=M_{f} / M_{0}=1$ and the reduced mass of high-charged particles is taken as $m_{v}=M_{v} / M_{0}=1,2,4,0.2$, and 0.5 , respectively. When $m_{f}=m_{v}=1$ (black squares) in Fig. 9(b), we are in the same case as in Fig. 8(a). As Fig. 9 shows, when $m_{v}>1$, the corresponding two curves (red dots and blue triangles) lie below the $m_{v}=1$ curve when $N<15$. Analogously, the other two curves with $m_{v}<1$ show higher LNF than the $m_{v}=1$ curve for $N<15$. Therefore we conclude that decreasing the mass of some particles can increase the LNF and increasing the mass leads to the opposite effect. This partially follows from the fact that dynamical matrix elements are inversely proportional to the square root of the particle mass [see Eq. (5)]. One should note that curves in Fig. 9 overlap for $N$ larger than a certain threshold number $N_{t}=14-16$. In the way we performed the simulation, we fixed the number of high-charged particles with mass $m_{v}$ and increased the number of low-charged particles with mass $m_{f}=1$. All particles of varied mass sit on the outer shell due to their higher charge, and for $N \geq N_{t}$, they do not participate in the LNF dynamic mode, which makes the effect of varying $m_{v}$ in Eq. (5) negligible. Obviously, $N_{t}$ can be shifted to higher values if the mass of a certain number of low-charged particles was changed as well.

\section{CONCLUSION}

The structural and spectral properties of classical, twodimensional binary clusters of differently charged particles in a hard-wall potential were investigated through modified Monte Carlo simulations. It was shown that the ground-state structure for a binary system mostly depends on the parameters: total number of particles $N$, number of high-charged particles $N_{v}$, and their charge $C_{r}$ compared to the charge of the remaining particles. The shell structure in our case is much more pronounced in comparison with the parabolic 

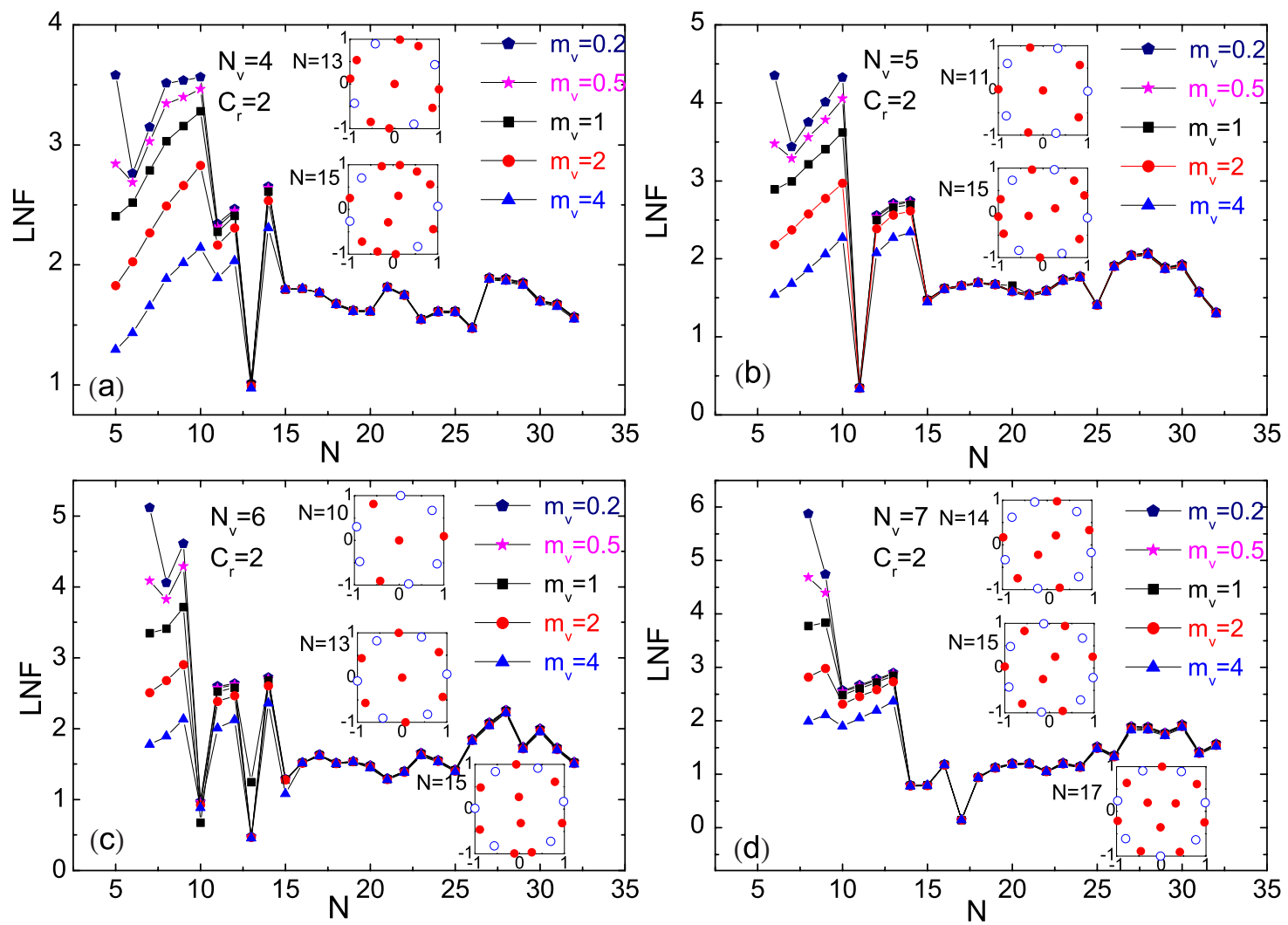

FIG. 9. (Color online) LNF as a function of the number of particles $N$ for clusters with $N_{v}=4-7, C_{r}=2, m_{f}=1$, and varying mass $m_{v}$ $=1,2,4,0.2,0.5$, respectively. The insets show typical particle configurations for some values of $N$.

case, and the high-charged particles always stay at the edge of the cluster, with low-charged particles distributed in between. The average energy per particle in the cluster was found to first decrease as a function of total number $N$ and increase for higher $N$, which is very different from the monotonic increase of $\bar{E}(N)$ found in monodisperse systems. Different values of $N_{v}$ and $C_{r}$ result in minimal interparticle interactions found for different numbers of particles in the cluster. As for structural novelties, we found that the critical number of particles in the inner shell for binary systems can be controlled by the values of $N_{v}$ and $C_{r}$, which was not the case in the monodisperse system. When $C_{r}$ is taken large enough, the highest number of particles in the second shell cannot exceed the number of high-charged particles $\left(N_{v}\right)$ before the third shell forms. This is completely different from monodisperse clusters in which the corresponding threshold number equals 6 .

The shell structures and observed structural properties have direct implications on the spectrum. Especially the ladder shape of maximal eigenfrequencies diagram $\omega_{\max }(N)$ shows the influence of cluster structure on the corresponding normal modes. Characteristic normal mode vectorplots were studied and discussed. The $\omega=0$ rotation-as-a-whole mode is found, together with a higher variety of BM- and CM-like modes compared to the monodisperse system. The latter are no more degenerate, and each have particular features, eigenfrequency and energy, caused by the asymmetric competing interactions in the system. As for the lowest nonzero fre- quency (LNF) mode, for monodisperse clusters it always corresponds to the intershell rotation. In the present case, for binary systems, because of the inhomogeneous spatial potential created by the two kinds of particles, the cluster can be excited in many different modes prior to intershell rotation. The LNF normal modes are determined by the ground-state configurations and demonstrate the essential influence of the inhomogeneous lateral confinement imposed by the outer shell on the inner particles in the cluster. On the one hand, the so-called matching structures, where the structure of the inner shell matches that of high-charged particles in the outmost shell, are stable and hard to be excited and on the other hand, the spatially homogeneous structures are less stable and easy to be excited. The latter can be reached for configurations with high-charged particles in the outer shell separated by equal numbers of low-charged particles, which we name uniform configurations. Those configurations exhibit the lowest LNFs. Finally, the effects of particle mass on the normal modes of binary systems are studied. As a general rule, we found that decreasing the mass of some particles leads to higher LNFs and vice versa.

\section{ACKNOWLEDGMENTS}

The authors would like to thank Dr. W. P. Ferreira for stimulating discussions. This work was supported by the bilateral project China-Flanders, the National Science Foundation of China under Grants No. 10504036 and No. 
90503005, the Special Funds for Major State Basic Research Project of China (973) under Grant No. 2005CB623603, Knowledge Innovation Program of Chinese Academy of Sciences, and Director Grants of CASHIPS. This work was partly supported by the SRF for ROCS, SEM. Part of the calculations were performed in the Center for Computational Science of CASHIPS and the Shanghai Supercomputer Center. M.V.M. acknowledges support from a Marie-Curie IntraEuropean Action. F.M.P. acknowledges support from the Flemish Science Foundation (FWO-Vl).
[1] E. P. Wigner, Phys. Rev. 46, 1002 (1934).

[2] C. C. Grimes and G. Adams, Phys. Rev. Lett. 42, 795 (1979).

[3] R. C. Ashoori, Nature (London) 379, 413 (1996).

[4] M. Golosovsky, Y. Saado, and D. Davidov, Phys. Rev. E 65, 061405 (2002).

[5] J. H. Chu and L. I, Phys. Rev. Lett. 72, 4009 (1994).

[6] G. A. Farias and F. M. Peeters, Solid State Commun. 100, 711 (1996).

[7] W. P. Ferreira, A. Matulis, G. A. Farias, and F. M. Peeters, Phys. Rev. E 67, 046601 (2003).

[8] V. M. Bedanov and F. M. Peeters, Phys. Rev. B 49, 2667 (1994).

[9] R. Bubeck, C. Bechinger, S. Neser, and P. Leiderer, Phys. Rev. Lett. 82, 3364 (1999).

[10] R. Bubeck, C. Bechinger, S. Neser, and P. Leiderer, Europhys. Lett. 60(3), 474 (2002).

[11] I. V. Schweigert, V. A. Schweigert, and F. M. Peeters, Phys. Rev. Lett. 84, 4381 (2000).

[12] M. Kong, B. Partoens, A. Matulis, and F. M. Peeters, Phys. Rev. E 69, 036412 (2004).

[13] M. E. Leunissen, C. G. Christova, A. P. Hynninen, C. P. Royall, A. I. Campbell, A. Imhof, M. Dijkstra, R. van Roij, and A. van Blaaderen, Nature (London) 437, 235 (2005).

[14] J. A. Drocco, C. J. Olson Reichhardt, C. Reichhardt, and B. Jankó, Phys. Rev. E 68, 060401(R) (2003).

[15] E. Yurtsever, F. Calvo, and D. J. Wales, Phys. Rev. E 72, 026110 (2005).

[16] K. Nelissen, B. Partoens, and F. M. Peeters, Phys. Rev. E 69, 046605 (2004).

[17] B. A. Grzybowski, X. Jiang, H. A. Stone, and G. M. White- sides, Phys. Rev. E 64, 011603 (2001).

[18] W. P. Ferreira, F. F. Munarin, K. Nelissen, R. N. Costa Filho, F. M. Peeters, and G. A. Farias, Phys. Rev. E 72, 021406 (2005).

[19] W. P. Ferreira, F. F. Munarin, G. A. Farias, and F. M. Peeters, J. Phys.: Condens. Matter 18, 9385 (2006).

[20] B. A. Grzybowski, H. A. Stone, and G. M. Whitesides, Nature (London) 405, 1033 (2000).

[21] L. Hornekær, N. Kjærgaard, A. M. Thommesen, and M. Drewsen, Phys. Rev. Lett. 86, 1994 (2001).

[22] Y. H. Liu, Z. Y. Chen, M. Y. Yu, L. Wang, and A. Bogaerts, Phys. Rev. E 73, 047402 (2006).

[23] T. Matthey, J. P. Hansen, and M. Drewsen, Phys. Rev. Lett. 91, 165001 (2003).

[24] K. Mangold, J. Birk, P. Leiderer, and C. Bechinger, Phys. Chem. Chem. Phys. 6, 1623 (2004).

[25] K. Nelissen, B. Partoens, I. Schweigert, and F. M. Peeters, Europhys. Lett. 74(6), 1046 (2006).

[26] V. A. Schweigert and F. M. Peeters, Phys. Rev. B 51, 7700 (1995).

[27] M. Kong, B. Partoens, and F. M. Peeters, Phys. Rev. E 65, 046602 (2002).

[28] I. V. Grigorieva, W. Escoffier, J. Richardson, L. Y. Vinnikov, S. Dubonos, and V. Oboznov, Phys. Rev. Lett. 96, 077005 (2006).

[29] L. R. E. Cabral, B. J. Baelus, and F. M. Peeters, Phys. Rev. B 70, 144523 (2004).

[30] A. Melzer, M. Klindworth, and A. Piel, Phys. Rev. Lett. 87, 115002 (2001).

[31] A. Melzer, Phys. Rev. E 67, 016411 (2003).

[32] D. J. Wales and A. M. Lee, Phys. Rev. A 47, 380 (1993). 